ARTICLE

\title{
High-yield, wafer-scale fabrication of ultralow-loss, dispersion-engineered silicon nitride photonic circuits
}

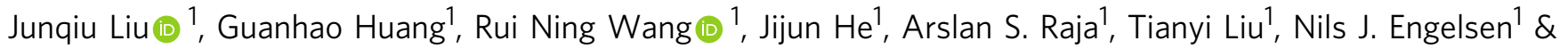
Tobias J. Kippenberg (1) ${ }^{1 凶}$

Low-loss photonic integrated circuits and microresonators have enabled a wide range of applications, such as narrow-linewidth lasers and chip-scale frequency combs. To translate these into a widespread technology, attaining ultralow optical losses with established foundry manufacturing is critical. Recent advances in integrated $\mathrm{Si}_{3} \mathrm{~N}_{4}$ photonics have shown that ultralow-loss, dispersion-engineered microresonators with quality factors $Q>10 \times 10^{6}$ can be attained at die-level throughput. Yet, current fabrication techniques do not have sufficiently high yield and performance for existing and emerging applications, such as integrated travelling-wave parametric amplifiers that require meter-long photonic circuits. Here we demonstrate a fabrication technology that meets all requirements on wafer-level yield, performance and length scale. Photonic microresonators with a mean $Q$ factor exceeding $30 \times$ $10^{6}$, corresponding to $1.0 \mathrm{~dB} \mathrm{~m}^{-1}$ optical loss, are obtained over full 4-inch wafers, as determined from a statistical analysis of tens of thousands of optical resonances, and confirmed via cavity ringdown with 19 ns photon storage time. The process operates over large areas with high yield, enabling 1-meter-long spiral waveguides with $2.4 \mathrm{~dB} \mathrm{~m}^{-1}$ loss in dies of only $5 \times 5 \mathrm{~mm}^{2}$ size. Using a response measurement self-calibrated via the Kerr nonlinearity, we reveal that the intrinsic absorption-limited $Q$ factor of our $\mathrm{Si}_{3} \mathrm{~N}_{4}$ microresonators can exceed $2 \times 10^{8}$. This absorption loss is sufficiently low such that the Kerr nonlinearity dominates the microresonator's response even in the audio frequency band. Transferring this $\mathrm{Si}_{3} \mathrm{~N}_{4}$ technology to commercial foundries can significantly improve the performance and capabilities of integrated photonics.

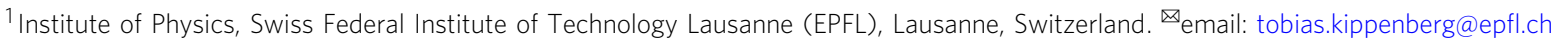


S ilicon photonics ${ }^{1,2}$ has evolved into a mature technology enabling the generation, modulation, and detection of optical signals on-chip, via heterogeneous or hybrid integration of different materials $s^{3-5}$. Many integrated devices have been demonstrated using silicon photonics, including siliconbased lasers 6,7 . Within the past two decades, they have been transferred from academic research to large-volume commercial deployment in datacenter interconnects. A second revolution is currently under way, where the optical nonlinearities of photonic integrated circuits (PICs) - accessed with continuous-wave lasers at sub-milliwatt power-can enable novel devices, of which Kerr frequency combs have been one of the studied processes. Dissipative Kerr soliton frequency combs ("soliton microcombs") ${ }^{8,9}$ constitute chip-scale frequency combs of broad bandwidths and repetition rates in the terahertz to microwave domain, and offer a route to highly compact frequency combs for a variety of applications in airborne systems or space. Microcombs are compatible with wafer-scale manufacturing as well as hybrid integration with III-V/Si lasers ${ }^{10-12}$, and have already been used in various system-level demonstrations including coherent telecommunications ${ }^{13,14}$, astronomical spectrometer calibration $^{15,16}$, ultrafast ranging ${ }^{17,18}$, massively parallel coherent $\operatorname{LiDAR}^{19}$, frequency synthesizers ${ }^{20}$, atomic clock architectures ${ }^{21}$, and neuromorphic computing 22,23 . Many integrated nonlinear photonic platforms for microcombs have emerged, ranging from $\mathrm{Si}_{3} \mathrm{~N}_{4}{ }^{24-30}$, diamond ${ }^{31}$, tantala ${ }^{32}$, and $\mathrm{SiC}^{33}$ to highly nonlinear $\mathrm{AlGaAs}^{34,35}$ and $\mathrm{GaP}^{36}$ on insulator, as well as electro-optic platforms such as $\mathrm{LiNbO}_{3}{ }^{37-40}$ and $\mathrm{AlN}^{41-43}$.

For integrated nonlinear photonics, in particular soliton microcombs, $\mathrm{Si}_{3} \mathrm{~N}_{4}{ }^{44}$ has emerged as a leading material due to its ultralow optical losses, absence of two-photon absorption in the telecommunication bands, strong Kerr nonlinearity, high refractive index, space compatibility ${ }^{45}$ and exceptionally high power handling capability ${ }^{46,47}$. To date, among all integrated photonic platforms ${ }^{48}$, optical losses near or below $1 \mathrm{~dB} \mathrm{~m}^{-1}$ have only been demonstrated in $\mathrm{Si}_{3} \mathrm{~N}_{4}$ PICs. While thin-core waveguides (i.e. waveguide height $h<100 \mathrm{~nm})^{49-51}$ have achieved ultralow losses, they are not suitable for nonlinear photonics due to the low effective refractive indices, low Kerr nonlinearity and large mode areas. Only recently, nonlinear, thick-core waveguides (i.e. $h>700 \mathrm{~nm})^{24-26}$ enabling negligible bending losses, dispersion engineering, and high Kerr nonlinearity, have been demonstrated, as outlined in Fig. 1. Most of the aforementioned system-level demonstrations of soliton microcombs ${ }^{13,15,17,19-21}$ are based on this type of $\mathrm{Si}_{3} \mathrm{~N}_{4}$ PICs. In addition, power-efficient supercontinuum generation has also been attained in the near-infrared as well as mid-infrared, enabling dualcomb spectrscopy $y^{52,53}$. Figure 1a highlights the lowest-loss thickcore (refs. ${ }^{24,25}$ and this work) and thin-core (refs. $\left.{ }^{49,50}\right) \mathrm{Si}_{3} \mathrm{~N}_{4}$ waveguides in terms of their optical losses and effective areas of the fundamental optical mode, in comparison with the state-of-the-art, lowest-loss silicon ${ }^{54}$, InP $\mathrm{P}^{55}$, and $\mathrm{AlGaAs}{ }^{35}$ waveguides. The tight confinement significantly reduces the bending loss, a key parameter for device footprint and photonic integration, as outlined in Fig. 1b. Though the desirable combination of tight confinement, ultralow loss, and engineered anomalous group-velocity dispersion (GVD) has been achieved ${ }^{24,25}$, it has only been attained in individual chips, i.e. with die-level throughput. Meanwhile, the fabrication of densely packed, meter-long PIC has not yet been realized, and neither has wafer-level fabrication yield, reliability and reproducibility, required for widespread adoption in CMOS foundries. Yet, densely packed, meter-long nonlinear $\mathrm{Si}_{3} \mathrm{~N}_{4}$ PIC could enable a new class of devices,

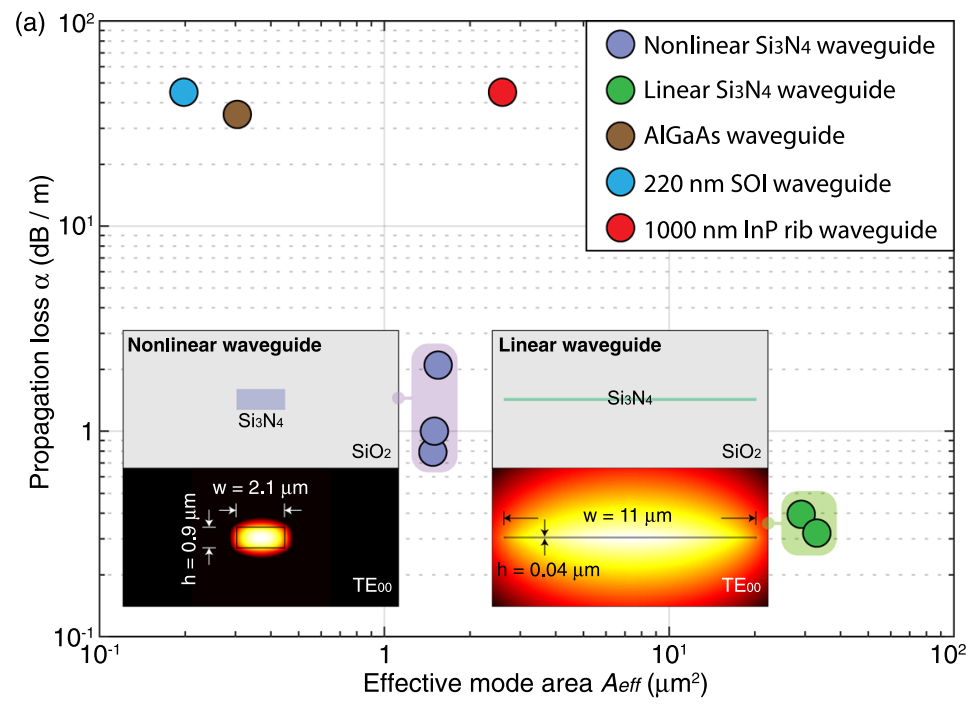

(b)
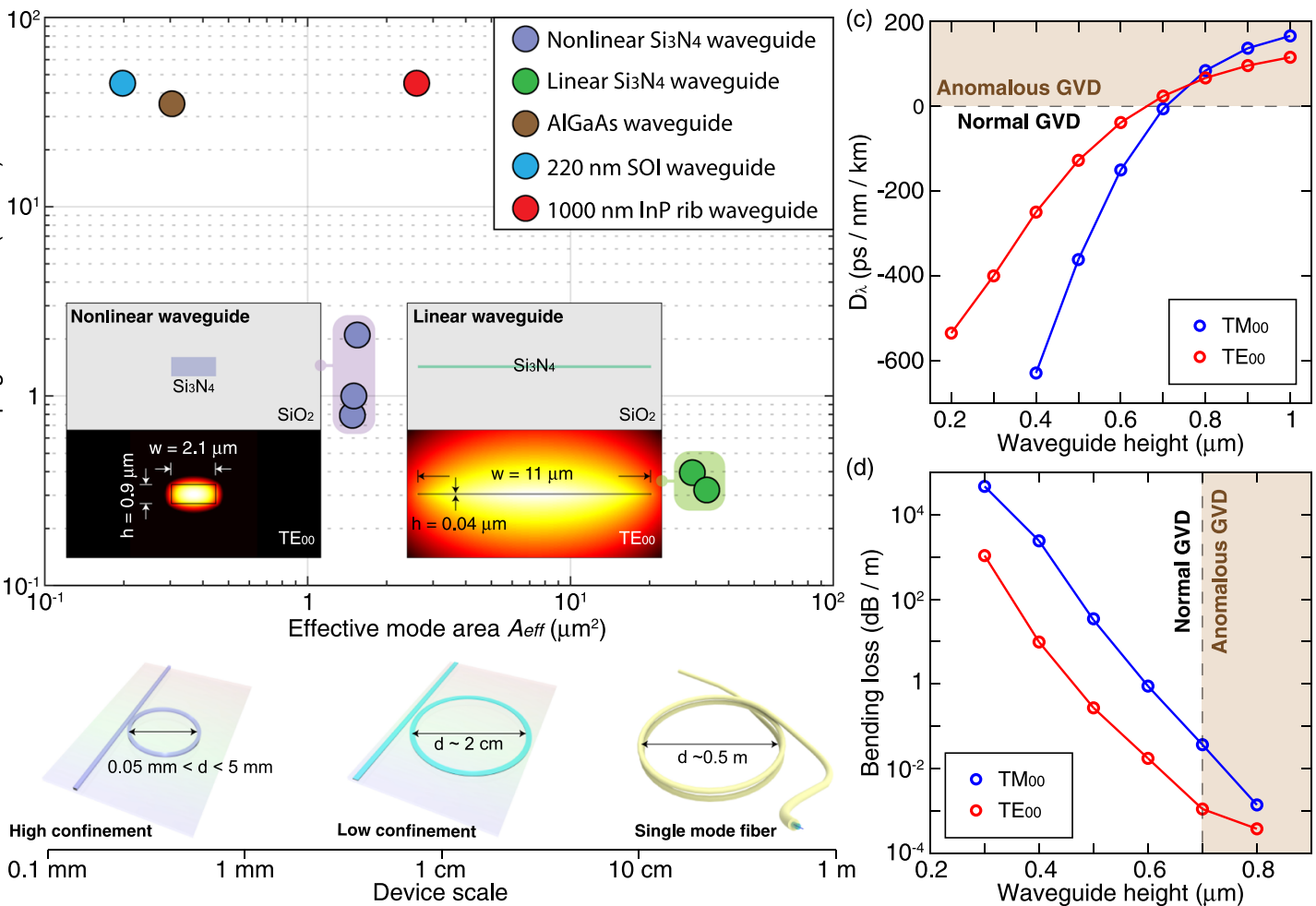

Fig. 1 Comparison of ultralow-loss linear and nonlinear $\mathrm{Si}_{\mathbf{3}} \mathbf{N}_{\mathbf{4}}$ platforms with state-of-the-art silicon, InP and AIGaAs platforms. a Comparison of optical losses and effective mode areas (in the telecommunication band of $1550 \mathrm{~nm}$ ) in state-of-the-art, low-loss waveguides, including nonlinear (i.e. thick-core, refs. 24,25 and this work) and linear (i.e. thin-core, refs. ${ }^{49,50}$ ) $\mathrm{Si}_{3} \mathrm{~N}_{4}$ waveguides, $220 \mathrm{~nm}$ silicon-on-insulator (SOI) waveguides ${ }^{54}, 1000 \mathrm{~nm}$ InP rib waveguides 55 , and nonlinear AIGaAs waveguides ${ }^{35}$. The insets show the waveguide geometry and optical mode profile of the $\mathrm{Si}_{3} \mathrm{~N}_{4}$ waveguides. $\mathbf{b}$ Comparison in device size for linear and nonlinear $\mathrm{Si}_{3} \mathrm{~N}_{4}$ waveguides and single-mode fibers. c Simulated GVD parameter $D_{\lambda}$ as a function of the waveguide height $h$, with a fixed waveguide width of $w=2.1 \mu \mathrm{m}$. d Simulated bending loss as a function of the waveguide height $h$, with a fixed bending radius of $d / 2=25 \mu \mathrm{m}$ and waveguide width of $w=2.1 \mu \mathrm{m}$. Anomalous-GVD region is brown-shaded, which is accessed with $h>700 \mathrm{~nm}$. The wavelength studied here is $1550 \mathrm{~nm}$. 
ranging from integrated traveling-wave parametric amplifiers ${ }^{56-59}$ to integrated mode-locked-lasers based on rare-earth doping ${ }^{60}$.

Here we report a high-yield, wafer-scale fabrication technology to build tight confinement, ultralow-loss, dispersion-engineered $\mathrm{Si}_{3} \mathrm{~N}_{4}$ waveguides of length exceeding one meter. This $\mathrm{Si}_{3} \mathrm{~N}_{4}$ fabrication technology is based on the photonic Damascene process $^{61}$ using standard CMOS fabrication techniques such as DUV stepper lithography, dry etching, and low-pressure chemical vapor deposition (LPCVD). Integrated $\mathrm{Si}_{3} \mathrm{~N}_{4}$ microresonators fabricated using this process are systematically characterized and analyzed, showing quality $(Q)$ factors above $30 \times 10^{6}$, linear losses of $1 \mathrm{~dB} \mathrm{~m}^{-1}$, and wafer-level yield.

\section{Results}

Wafer-scale-yield photonic Damascene process. Figure 2a shows the process flow of the optimized photonic Damascene process, and scanning electron micrographs (SEM) for selected key steps. The waveguides and stress-release filler patterns ${ }^{61,62}$ are written directly on the $\mathrm{SiO}_{2}$ substrate via DUV stepper lithography based on a $248 \mathrm{~nm} \mathrm{KrF}$ excimer laser. The use of DUV, in contrast to the commonly employed electron-beam lithography, enables a dramatic increase in fabrication throughput, stability, and reproducibility, essential to large-volume manufacturing. The patterns are then dry-etched into the $\mathrm{SiO}_{2}$ substrate to create waveguide preforms. We note that our $\mathrm{SiO}_{2}$ dry etching does not introduce a trade-off between the etch verticality and surface roughness. Figure $2 \mathrm{~d}$ top shows the sidewall bottom angle $90^{\circ}<\beta$ $<92^{\circ}$. To further reduce the waveguide's root mean square sidewall roughness to sub-nanometer level, the substrate is annealed at $1250^{\circ} \mathrm{C}$ ("preform reflow") ${ }^{63}$. Importantly, this reflow process can further reduce the scattering loss (see Supplementary Information), and does not lead to significant deformation of the waveguide preform. Figure $2 \mathrm{~d}$ bottom shows the measured sidewall bottom angle $\beta \approx 93^{\circ}$, after reflow. An LPCVD $\mathrm{Si}_{3} \mathrm{~N}_{4}$ film of $1000 \mathrm{~nm}$ thickness is deposited on the patterned substrate, filling the preform trenches and forming the waveguides. To improve the yield and allow wafer-scale processing, an etchback planarization process is applied (see Supplementary Information), combining photoresist coating, dry etching and chemical-mechanical planarization (CMP). This process enables full control of the polishing depth, sub-nanometer surface roughness, and wafer-scale uniformity of $\mathrm{Si}_{3} \mathrm{~N}_{4}$ waveguide height with variation below $3 \%$, critical for monolithic or heterogeneous integration of piezoelectric materials ${ }^{64,65}$, electro-optic materials $^{66}$, and monolayer two-dimensional materials ${ }^{67}$. Afterward, the substrate is thermally annealed at $1200^{\circ} \mathrm{C}$ to drive out the residual hydrogen impurities in the $\mathrm{Si}_{3} \mathrm{~N}_{4}$ film 26,68 . A top $\mathrm{SiO}_{2}$ cladding composed of TEOS and low-temperature oxide is deposited on the wafer, followed by thermal annealing of the $\mathrm{SiO}_{2}$ at $1200^{\circ} \mathrm{C}$. Finally, the wafer is separated into chips via deep dry etching followed by dicing or backside grinding, to attain chip facets with the superior quality required for edge coupling ${ }^{11,12}$.

Figure $2 \mathrm{~b}$ shows the final $\mathrm{Si}_{3} \mathrm{~N}_{4}$ chips which contain multiple ring resonators of different free spectral ranges (FSRs). Figure $2 \mathrm{c}$ shows the optical micrograph of the $\mathrm{Si}_{3} \mathrm{~N}_{4}$ microring resonator, bus waveguide, and filler patterns, as well as the tightly confined waveguide mode. The resulting negligible bending loss allows high- $Q$ microresonators of small radii (below $23 \mu \mathrm{m}$, i.e. $1 \mathrm{THz}$ FSR), which find widespread applications in optical filters and coupled-resonator-based delay lines ${ }^{69,70}$. The filler patterns consist of horizontal and vertical bars uniformly distributed over the entire wafer area, and can significantly relax the as-deposited LPCVD $\mathrm{Si}_{3} \mathrm{~N}_{4}$ film stress for crack prevention (see Supplementary Information). These filler patterns are also required for etching and CMP uniformity. In terms of the number of usable chips free from cracks, our fabrication yield is $100 \%$, as no cracks have been observed in the past runs of more than 30 wafers using the same stress-release filler patterns. (a) 1. Lithography
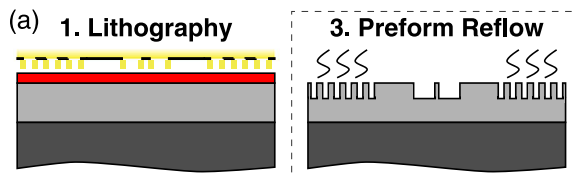

2. Dry Etching
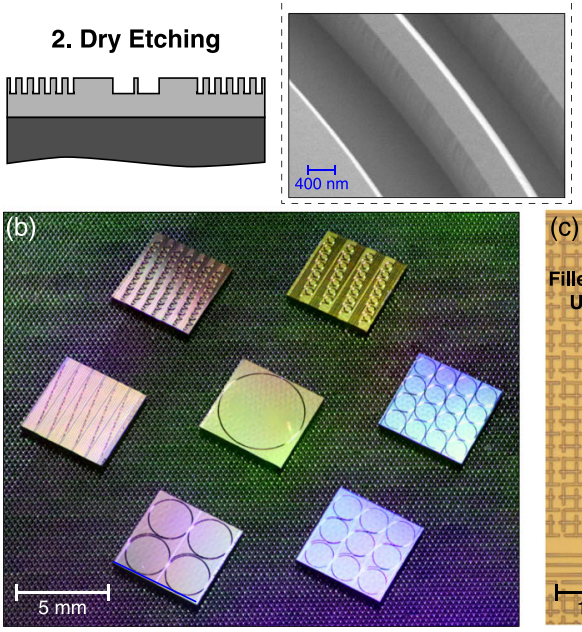

4. Film Deposition
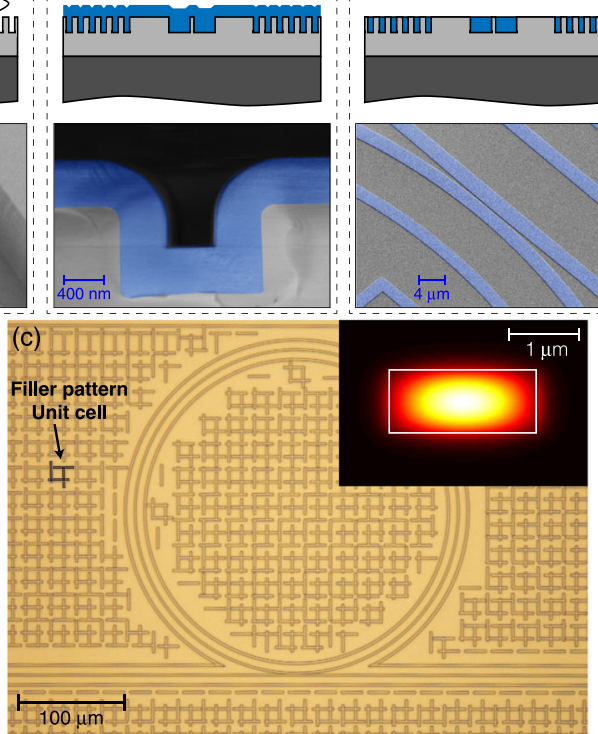

5. Planarization

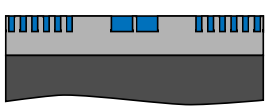

6. Cladding Deposition
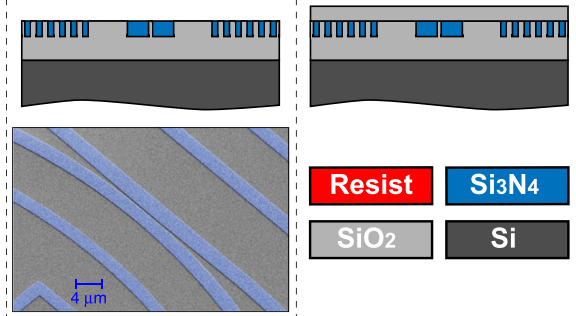

$\mathrm{SHO}_{2}$
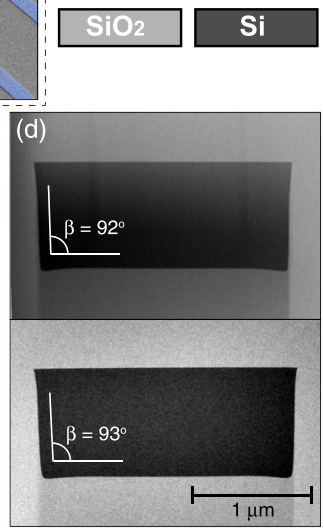

Fig. 2 The wafer-scale photonic Damascene process flow and highlighted features. a Process flow of the optimized photonic Damascene process including DUV stepper lithography, preform dry etching, preform reflow, LPCVD $\mathrm{Si}_{3} \mathrm{~N}_{4}$ deposition, planarization, and $\mathrm{SiO}_{2}$ cladding deposition. The blue shaded parts are $\mathrm{Si}_{3} \mathrm{~N}_{4}$. b Photograph showing $\mathrm{Si}_{3} \mathrm{~N}_{4}$ photonic chips with microring resonators of different FSRs. c Optical micrograph showing the bus waveguide, microring resonator, and filler patterns (used to prevent crack formation). Inset: simulated tightly confined optical mode. d Transmission electron micrographs (TEM) of the waveguide cross-sections, before (top) and after (bottom) the preform reflow. The reflow preserves the waveguide dimensions accurately, while removing high-frequency spatial roughness. 

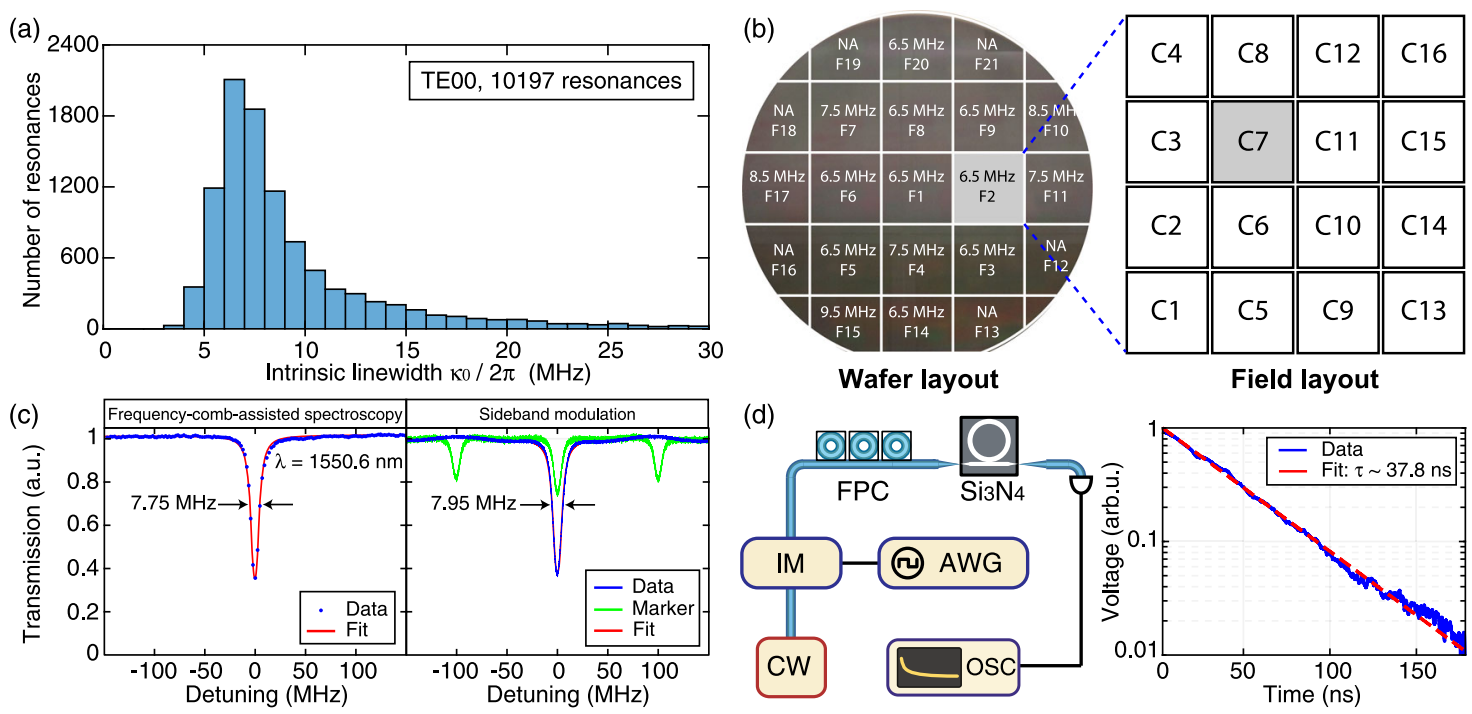

Fig. 3 Statistical study of microresonator $\mathbf{Q}$ factors using multiple techniques. a Histogram of 10,197 TE showing the most probable value of $\kappa_{0} / 2 \pi=6.5 \mathrm{MHz}$ and $Q_{0}=30 \times 10^{6}$. b DUV stepper exposure layout, and the most probable value $\kappa_{0} / 2 \pi$ of the C7 chips at different positions on the wafer. The reticle design containing sixteen chips is uniformly exposed in discrete fields over a 4-inch wafer. NA: not applicable, due to visible photoresist coating defects or missing C7 chips near the wafer edge. $\mathbf{c}$ Linewidth measurement of the same resonance at 1550.6 $\mathrm{nm}$ using frequency-comb-assisted diode laser spectroscopy (left, $\kappa / 2 \pi=7.75 \mathrm{MHz}$ and $\kappa_{0} / 2 \pi=5.87 \mathrm{MHz}$ ) and sideband modulation technique (right, $\kappa$ / $2 \pi=7.95 \mathrm{MHz}$ and $\kappa_{0} / 2 \pi=6.05 \mathrm{MHz}$ ). This resonance does not present a visible mode split. $\mathbf{d}$ Cavity ring-down measurement. An intensity modulator (IM) is used to switch off the pump field. The cavity ring-down signal is averaged 1000 times. The exponential fit gives an optical field decay time of $\tau=$ $37.8 \mathrm{~ns}$, corresponding to a photon decay time of $18.9 \mathrm{~ns}$ and a loaded linewidth of $\kappa / 2 \pi=8.4 \mathrm{MHz}$. arb.u: arbitrary unit, AWG: arbitrary function generator, OSC: oscilloscope, CW: continuous-wave laser, FPC: fiber polarization controller.

Statistical analysis of microresonator quality factors. We fabricate $\mathrm{Si}_{3} \mathrm{~N}_{4}$ microresonators of $40 \mathrm{GHz}$ FSR, $2200 \mathrm{~nm}$ width and $950 \mathrm{~nm}$ height, and systematically study the microresonator $Q$ factors (i.e. losses). Frequency-comb-assisted diode laser spectroscopy ${ }^{26,71}$ is used to characterize the resonance frequency $\omega_{\text {opt }} / 2 \pi$ and linewidth $\kappa / 2 \pi$, which relate to the resonance $Q$ factor as $Q=\omega_{\text {opt }} / \kappa$. Here we mainly study the fundamental transverse electric $\left(\mathrm{TE}_{00}\right)$ mode. The total (loaded) linewidth $\kappa / 2 \pi=\left(\kappa_{0}+\kappa_{\mathrm{ex}}\right) / 2 \pi$, the intrinsic loss $\kappa_{0} / 2 \pi$ and the coupling strength $\kappa_{\mathrm{ex}} / 2 \pi$ are extracted from each resonance fit. Figure 3a shows the $\kappa_{0} / 2 \pi$ histogram of $10,197 \mathrm{TE}_{00}$ resonances measured from twenty-six microresonators. The most probable value is $\kappa_{0} / 2 \pi=6.5 \mathrm{MHz}$, corresponding to an intrinsic $Q$ factor of $Q_{0}=$ $30 \times 10^{6}$. In comparison, $\kappa_{0} / 2 \pi=9.5 \mathrm{MHz}$ is found for the fundamental transverse magnetic $\left(\mathrm{TM}_{00}\right)$ mode, corresponding to $Q_{0}=20 \times 10^{6}$ (see Supplementary Information). In addition, by plotting the fitted $\kappa_{0} / 2 \pi$ as a function of the resonance wavelength measured from $1260 \mathrm{~nm}$ to $1630 \mathrm{~nm}$, a weak trend showing a larger $\kappa_{0} / 2 \pi$ at a shorter wavelength is observed (see Supplementary Information). Finally, as the threshold power for soliton formation scales as $1 / Q^{2}$, such a high microresonator $Q$ allows soliton formation with $40 \mathrm{GHz}$ repetition rate with only $10 \mathrm{~mW}$ optical power, obviating the need for an optical power amplifier (see Supplementary Information).

Next, we demonstrate the wafer-scale yield of our fabrication technology. Figure $3 b$ shows our mask layout comprising $4 \times$ 4 chip designs on the DUV stepper reticle. Each chip has a $5 \times$ $5 \mathrm{~mm}^{2}$ size, and contains multiple microresonators as shown in Fig. 2b. The DUV stepper writes the reticle pattern uniformly over the full 4-inch wafer in discrete fields. The calibration chips of $40 \mathrm{GHz}$ FSR studied here are the $\mathrm{C} 7$ chips. The most probable values of $\kappa_{0} / 2 \pi$ for the C7 chips are measured and plotted in each exposure field, as shown in Fig. $3 \mathrm{~b}$ (see each individual histogram in Supplementary Information). In most fields, $\kappa_{0} / 2 \pi \leqslant 7.5 \mathrm{MHz}$ is found. While exceptionally narrow linewidths have been reported previously in individual resonances, our statistics based on tens of thousands of analyzed resonances from dozens of samples at different wafer positions shows wafer-scale fabrication throughput and yield. In terms of loss values or microresonator $Q$ factors, we define the yield as the ratio between the number of the chips with linewidths below $20 \mathrm{MHz}$ (i.e. $Q_{0}>10 \times 10^{6}$ ) to the total number of measured chips. In the present case of $40-\mathrm{GHz}$ FSR chips, the yield is calculated as $15 / 17=89 \%$ (The C7 chips in F12 and F13 are not available because of visible lithography defects; the C7 chips in F16, F18, F19, F21 are partially exposed, thus these four are not counted). In the Supplementary Information, the wafer-scale loss measurement is also illustrated on another wafer containing $10-\mathrm{GHz}-\mathrm{FSR}$ chips (used in ref. ${ }^{72}$ ). Besides, a statistical process analysis based on fifteen wafers is shown, demonstrating the wafer-to-wafer reproducibility of our fabrication process.

In addition, a sideband modulation technique ${ }^{73}$ is used to measure the resonance linewidth $\kappa / 2 \pi$ and to fit $\kappa_{0} / 2 \pi$. Two sidebands, each separated from the carrier by $100 \mathrm{MHz}$, are used to calibrate the resonance linewidth. Figure $3 c$ compares the measured $\kappa / 2 \pi$ and fitted $\kappa_{0} / 2 \pi$ of the same resonance that does not present a visible mode split, using both the frequency-combassisted diode laser spectroscopy $\left(\kappa / 2 \pi=7.75 \mathrm{MHz}\right.$ and $\kappa_{0} / 2 \pi=$ $5.87 \mathrm{MHz})$ and the sideband modulation technique $(\kappa / 2 \pi=7.95$ $\mathrm{MHz}$ and $\kappa_{0} / 2 \pi=6.05 \mathrm{MHz}$ ). Both methods agree with each other, and show $Q_{0}>32 \times 10^{6}$.

Furthermore, a cavity ring-down measurement is performed to validate the measured linewidth (see Methods). Figure $3 \mathrm{~d}$ shows the schematic of the experimental setup and a representative ringdown measurement data. The fitted optical field decay time is $37.8 \mathrm{~ns}$, corresponding to $18.9 \mathrm{~ns}$ photon storage time. The calculated loaded linewidth is $\kappa / 2 \pi=8.4 \mathrm{MHz}$, showing consistency between the three characterization methods used here.

Meter-long spiral waveguides. In addition to high- $Q$ microresonators, we also fabricate and characterize meter-long spiral 

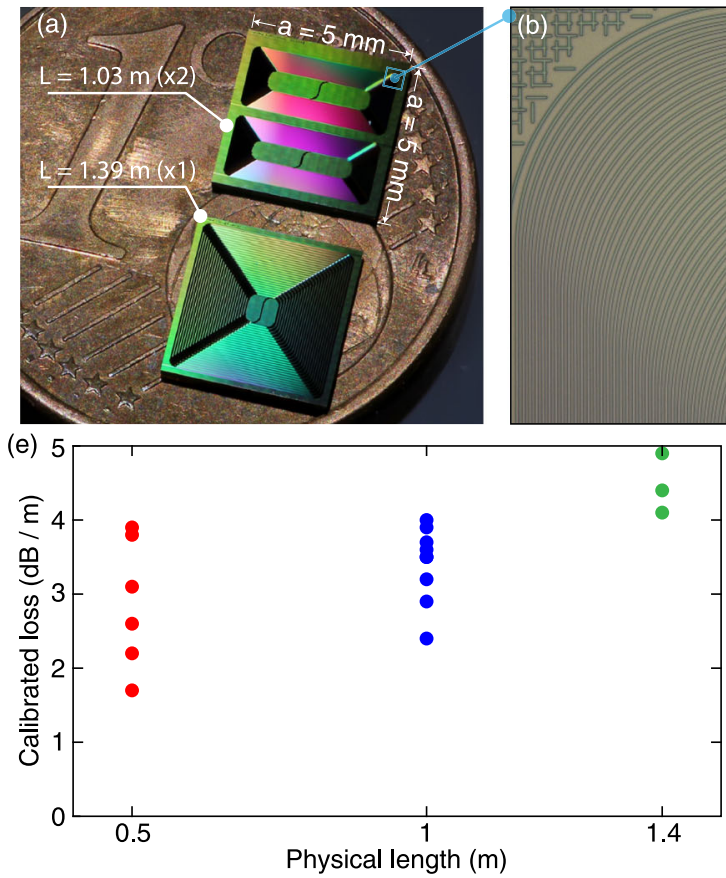
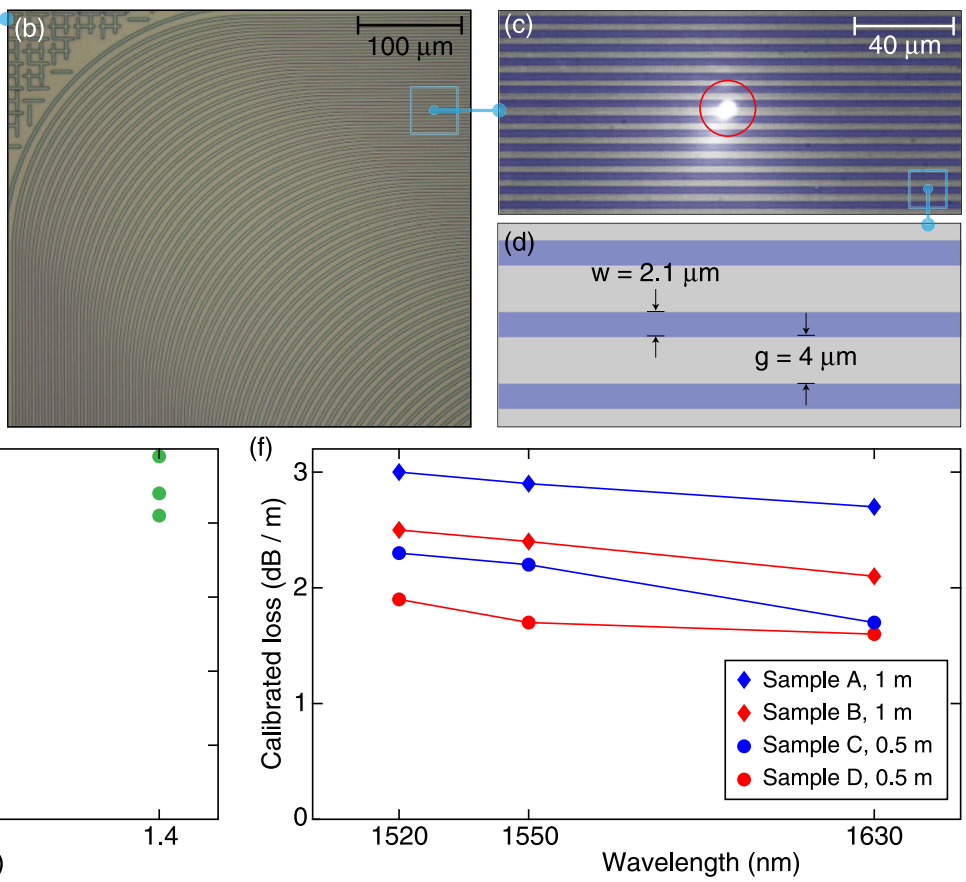

Fig. 4 Small-footprint, meter-long, ultralow-loss $\mathbf{S i}_{\mathbf{3}} \mathbf{N}_{\mathbf{4}}$ spiral waveguides. a Photograph showing $\mathrm{Si}_{3} \mathrm{~N}_{4}$ chips containing two 1.0-meter-long and one 1.4meter-long spiral waveguides. b, c Optical micrographs of the densely packed $\mathrm{Si}_{3} \mathrm{~N}_{4}$ waveguides in Archimedean spirals, taken with a yellow light camera (b) and IR camera (c). When $1550 \mathrm{~nm}$ laser light is coupled into the waveguide, light-scattering defects are observed under the IR camera (highlighted with a red circle). d Schematic showing the waveguide width and spacing. e Measured and calibrated optical losses at $1550 \mathrm{~nm}$ wavelength in $0.5 \mathrm{~m}, 1.0 \mathrm{~m}$, and $1.4 \mathrm{~m}$ long spiral waveguides. f Measured and calibrated losses at different wavelengths for four selected samples.

waveguides that are key elements to build photonic true-time delay lines. Previously, silica suspended wedge waveguides ${ }^{74}$ and thin-core $\mathrm{Si}_{3} \mathrm{~N}_{4}$ waveguides ${ }^{51}$ have been studied to build delay lines with losses below $0.1 \mathrm{~dB} \mathrm{~m}^{-1}$. However, in order to avoid bending losses, these waveguides must occupy more than $20 \mathrm{~cm}^{2}$ areas, thus requiring significant device footprints. While tight optical confinement can reduce the footprint, losses approaching even $1 \mathrm{~dB} \mathrm{~m}^{-1}$ have not been achieved in any nonlinear waveguide including thick-core $\mathrm{Si}_{3} \mathrm{~N}_{4}$. Here, we demonstrate meterlong $\mathrm{Si}_{3} \mathrm{~N}_{4}$ waveguides featuring ultralow loss and small footprint, which can enable key applications such as traveling-wave parametric amplifiers ${ }^{56-59}$, rare-earth-doped mode-locked lasers ${ }^{60}$ and optical coherence tomography $(\mathrm{OCT})^{75}$.

Figure 4a shows a photograph of photonic chips containing $\mathrm{Si}_{3} \mathrm{~N}_{4}$ waveguides of physical lengths $L>1 \mathrm{~m}$. Figure $4 \mathrm{~b}-\mathrm{d}$ shows the spiral layout. The waveguides are densely packed in Archimedean spirals, with waveguide width $w=2.1 \mu \mathrm{m}$ and gap distance $g=4 \mu \mathrm{m}$. Three lengths are studied here: a 0.5 -meterlong spiral containing 50 coils and covering a $3.1 \mathrm{~mm}^{2}$ area; a 1.0 meter-long spiral containing 106 coils and covering a $6.6 \mathrm{~mm}^{2}$ area; and a 1.4-meter-long spiral containing 130 coils and covering a $20.2 \mathrm{~mm}^{2}$ area. Compared with the previous work based on thin-core $\mathrm{Si}_{3} \mathrm{~N}_{4}$ waveguides ${ }^{51}$ showing a device footprint of more than $20 \mathrm{~cm}^{2}$ area for 1 meter physical length, our devices reduce the necessary footprint by a factor of 300, critical for photonic integration. Figure $4 \mathrm{e}$ shows the measured losses in multiple samples, calibrated using the adjacent 5-millimeter-long waveguide which has a fiber-chip-fiber through coupling efficiency of $33 \%$ ( $4.8 \mathrm{~dB}$ for two chip facets). The lowest-loss values found are $1.7 \mathrm{~dB} \mathrm{~m}^{-1}$ for $0.5 \mathrm{~m}$ length, $2.4 \mathrm{~dB} \mathrm{~m}^{-1}$ for 1.0 $\mathrm{m}$ length, and $4.1 \mathrm{~dB} \mathrm{~m}^{-1}$ for $1.4 \mathrm{~m}$ length. These loss values are higher than the value extrapolated from microresonator $Q$ characterization $\left(1.0 \mathrm{~dB} \mathrm{~m}^{-1}\right)$. Meanwhile, the overall trend shows higher losses in longer waveguides. We attribute both observations to additional light-scattering defects. Light- scattering defects are found under an infrared (IR) microscope, as shown in Fig. 4c. By counting the number of defects in highloss spirals, we estimate that each defect causes 1-2 dB extra loss. The defect probability depends on the waveguide area. These defects are likely caused by particle contamination on the wafer, as we have verified that these defects are not on the DUV reticle which would generate the same defects in the same position in each exposure field. Figure $4 \mathrm{f}$ shows the calibrated losses measured at different wavelengths for four selected samples. Again, a trend showing higher losses at shorter wavelengths is observed.

Quantitative analysis of absorption losses using a Kerrcalibrated linear response measurement. Next, we quantitatively investigate the intrinsic absorption and scattering losses of our $\mathrm{Si}_{3} \mathrm{~N}_{4}$ waveguides. The optical losses in the telecommunication band have two main contributions: Rayleigh scattering losses that are mainly caused by waveguide sidewall roughness, and absorption losses due to e.g. hydrogen impurities. While the hydrogen absorption loss can be efficiently eliminated via repeated thermal annealing of $\mathrm{Si}_{3} \mathrm{~N}_{4}$ at high temperature $\left(\sim 1200^{\circ} \mathrm{C}\right)^{26,68}$, efforts on loss reduction have mainly been focused on reducing waveguide roughness via optimized dry etching ${ }^{25}$ and etchless processes ${ }^{76,77}$. In addition, the large mode area of thin-core $\mathrm{Si}_{3} \mathrm{~N}_{4}$ waveguides ${ }^{49-51}$ results in reduced optical mode interaction with waveguide sidewall roughness, and thereby reduced scattering losses.

To quantify the thermal absorption loss of our $\mathrm{Si}_{3} \mathrm{~N}_{4}$ waveguides, a linear response measurement ${ }^{36}$ is performed. We characterize the resonance frequency shift response of the probe mode induced by intensity modulation of the pump mode, and utilize the measured response to calibrate the absorption loss of the pump resonance. The method exploits the fact that the thermal response $\chi_{\text {therm }}(\omega)$ dominates at low frequency, while the Kerr response $\chi_{\text {Kerr }}(\omega)$ dominates at higher frequency. The experimental setup is shown in Fig. 5a. The pump laser is first 
(a)

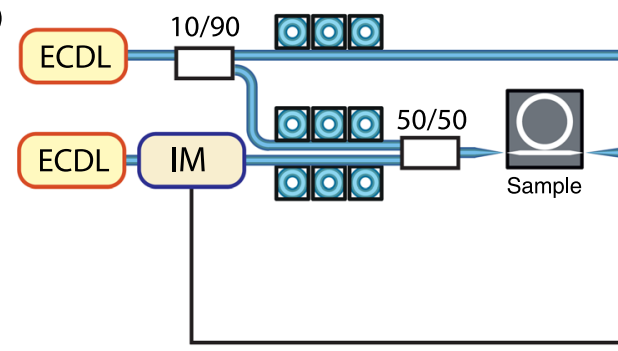

(c)

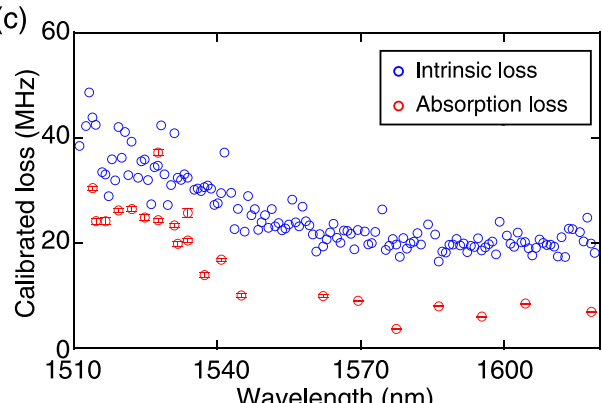

(e)

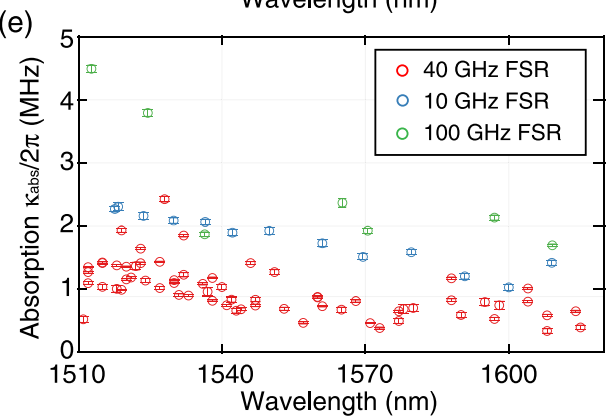

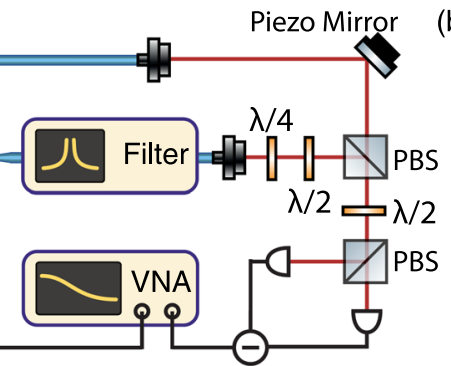

(b)

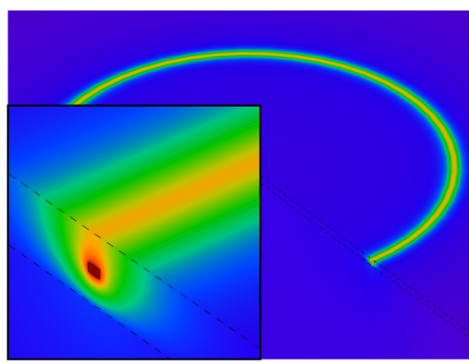

(d)
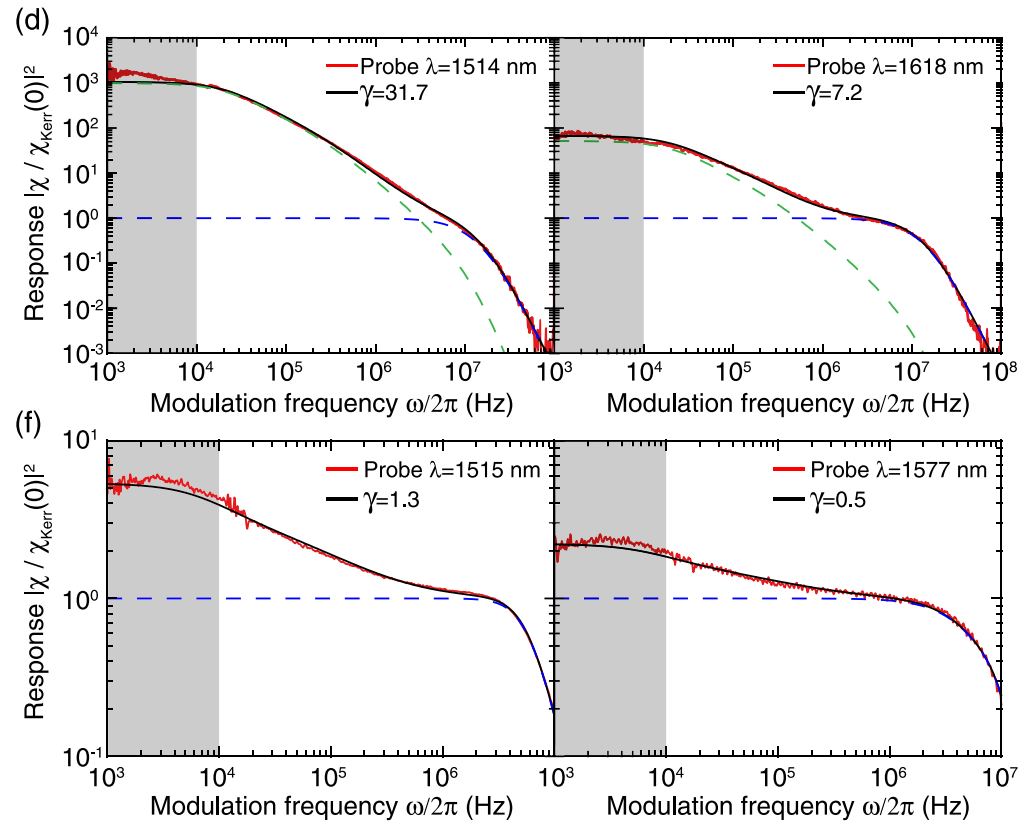

Fig. 5 Probing the absorption loss of $\mathrm{Si}_{3} \mathbf{N}_{\mathbf{4}}$ microresonators via Kerr-nonlinearity-calibrated thermal response measurements. a Experimental setup. ECDL: external-cavity diode lasers. IM: intensity modulator. VNA: vector network analyzer. PBS: polarization beam splitter. $\mathbf{b}$ Thermal simulation of the temperature distribution in the waveguide structures. c Comparison of the loss values measured using the response measurement and frequency-combassisted diode laser spectroscopy, on a partially annealed sample with prominent hydrogen absorption losses. This comparison reveals an approximate, wavelength-independent, $12 \mathrm{MHz}$ loss difference between the two datasets, which is presumed to be due to intrinsic scattering loss. $\mathbf{d}$ For the two resonances at $1514 \mathrm{~nm}$ and $1618 \mathrm{~nm}$ shown in (c), the measured (red) and fitted (black) frequency response $\chi(\omega)$ normalized to $\chi_{\text {Kerr }}$. The fitted cavity cutoff frequencies are, $\kappa / 4 \pi=22.7 \mathrm{MHz}(\lambda=1514 \mathrm{~nm})$, and $\kappa / 4 \pi=17.1 \mathrm{MHz}(\lambda=1618 \mathrm{~nm})$. e Calibrated absorption loss $\kappa_{\mathrm{abs}} / 2 \pi$ of different resonances from different samples. f For the two resonances at $1515 \mathrm{~nm}$ and $1577 \mathrm{~nm}$ of the $40-\mathrm{GHz}-\mathrm{FSR}$ samples shown in (e), the measured frequency response $\chi(\omega)$ normalized to $\chi_{\text {Kerr }}$ (red and black). The fitted cavity cutoff frequencies are, $\kappa / 4 \pi=7.4 \mathrm{MHz}(\lambda=1515 \mathrm{~nm})$, and $\kappa / 4 \pi=10.8 \mathrm{MHz}(\lambda=1577 \mathrm{~nm})$. In $(\mathbf{d}, \mathbf{f})$, we show the fitted Kerr (blue) and thermal (green) responses, and the gray areas mark the modulation frequency range from 1 to $10 \mathrm{kHz}$ (the measured response within this range is not included in the response fitting). The fitted values of $\gamma=\frac{\chi_{\text {therr }}(0)}{\chi_{\text {Kerr }}(0)}$ are shown in $(\mathbf{d}, \mathbf{f})$. The fitted thermal response $\left|\frac{\chi_{\text {therm }}(\omega)}{\chi_{\text {Kerr }}(0)}\right|^{2}$ is not shown in (f) as it is mostly below $10^{-1}$. The error bars shown in (c, e) account for fitting errors ( $95 \%$ confidence interval).

tuned to the optical resonance (frequency $v_{m}$ ) for which the thermal absorption loss $\kappa_{\mathrm{abs}} / 2 \pi$ is to be characterized, and is then intensity-modulated at a frequency of $\omega / 2 \pi$ which is swept from $1 \mathrm{kHz}$ to $100 \mathrm{MHz}$. Meanwhile, the probe laser is loosely locked (i.e. with $\sim 300 \mathrm{~Hz}$ locking bandwidth) to a probe resonance whose frequency is $\nu^{\prime}$.

This intensity modulation causes a change in the intracavity photon number $n_{c}$ of the pump mode, which modulates the resonance frequency of the probe mode via Kerr and thermal nonlinearities. The pump power is maintained sufficiently low $\mathcal{O}(100 \mu \mathrm{W})$, such that the steady-state frequency shift of the probe mode is small compared to the resonance linewidth $\kappa / 2 \pi$, i.e. $\delta \nu_{m^{\prime}} \ll \kappa / 2 \pi$. In this linear regime, the probe mode's frequency response to the modulated pump power is given by ${ }^{36}$

$$
\chi(\omega)=\frac{\delta \nu_{m^{\prime}}}{\delta n_{\mathrm{ph}}}=\chi_{\text {therm }}(\omega)+\chi_{\text {Kerr }}(\omega) .
$$

The total response $\chi(\omega)$ consists of two parts: the Kerr response $\chi_{\text {Kerr }}(\omega)$ with infinite bandwidth, and the thermal response $\chi_{\text {therm }}(\omega)$ with a corner frequency around $40 \mathrm{kHz}$. Therefore, by calibrating the response $\chi(\omega)$ as a function of the modulation frequency $\omega / 2 \pi, \chi_{\text {therm }}(\omega)$ and $\chi_{\text {Kerr }}(\omega)$ can be individually identified. Using the inferred values of $\chi_{\text {therm }}(\omega)$ and $\chi_{\text {Kerr }}(\omega)$ at DC $(\omega=0)$, the absorption rate is calculated as

$$
\kappa_{\mathrm{abs}}=\frac{2 c n_{\mathrm{mat}} n_{2}}{n_{g} n_{\mathrm{eff}} V_{\text {eff }} \frac{\mathrm{d} T}{\mathrm{~d} P_{\mathrm{abs}}} \frac{\mathrm{d} n_{\text {mat }}}{\mathrm{d} T}} \frac{\chi_{\text {therm }}(0)}{\chi_{\text {Kerr }}(0)}
$$

where $V_{\text {eff }}$ is the effective optical mode volume, $n_{2}=2.4 \times 10^{-19} \mathrm{~m}^{2} /$ $\mathrm{W}$ is the nonlinear index of $\mathrm{Si}_{3} \mathrm{~N}_{4}, n_{g}=2.1$ is the group index, $n_{\text {mat }}$ $=2.0$ is the material index, $n_{\text {eff }}=1.8$ is the effective index, $\mathrm{d} n_{\text {mat }} /$ $\mathrm{d} T=2.5 \times 10^{-5} / \mathrm{K}$ is the thermo-optic coefficient of $\mathrm{Si}_{3} \mathrm{~N}_{4}$, and $P_{\mathrm{abs}}$ is the absorbed power. The full derivation of Eq. (2) is shown in the Supplementary Information.

The frequency response $\delta \nu_{m^{\prime}}$ to the pump modulation is transduced into phase modulation of the probe laser. This phase modulation is measured using balanced homodyne detection, with the pump laser filtered out before detection (see "Methods"). To evaluate the absorption rate $\kappa_{\mathrm{abs}}$, the factor $\gamma=\chi_{\text {therm }}(0) /$ 
$\chi_{\text {Kerr }}(0)$ is retrieved by fitting the response function shown in Eq. (3) in "Methods", which consists of one simulated thermal cutoff and two cavity cutoffs, to the measured response at frequencies above $10 \mathrm{kHz}$. This $10 \mathrm{kHz}$ frequency cutoff for fitting is chosen to be far outside the locking bandwidth, and the thermal response simulation within this frequency range is still validated. Therefore, we actually anchor the response DC offset in Eq. (2) at 10 $\mathrm{kHz}$. Finite-element simulations of optical mode profiles and bulk absorption heating (see Fig. 5b) are performed to calculate the coefficients $V_{\text {eff }}$ and to retrieve the frequency-domain thermal response function $\frac{\mathrm{d} T}{\mathrm{~d} P_{\text {abs }}}(\omega)$ used in the fitting (see "Methods"). The simulation model we use to extract the thermal response function is validated by our recent measurement ${ }^{78}$ of the thermorefractive noise in these $\mathrm{Si}_{3} \mathrm{~N}_{4}$ devices. Figure $5 \mathrm{~d}, \mathrm{f}$ presents four examples of measured and fitted $\chi(\omega)$. Supplementary Information provides more details on data analysis.

To validate our method, we first benchmark the linear response measurement by characterizing a partially annealed $\mathrm{Si}_{3} \mathrm{~N}_{4}$ sample whose resonance linewidth data have been published in ref. ${ }^{26}$. We characterize this particular sample again, using both the response measurement and frequency-comb-assisted diode laser spectroscopy, and compare the results using both methods in Fig. 5c. Assuming a wavelength-independent scattering loss of 12 $\mathrm{MHz}$, the measured absorption loss using the response measurement agrees with the total loss measured spectroscopically.

Figure $5 \mathrm{e}$ shows the calculated absorption rates $\kappa_{\mathrm{abs}} / 2 \pi$ of different resonances from four $40-\mathrm{GHz}-\mathrm{FSR} \mathrm{Si}_{3} \mathrm{~N}_{4}$ samples featuring $Q_{0}>30 \times 10^{6}$ (this work), in comparison with 10GHz-FSR samples (used in ref. ${ }^{72}, \kappa_{0} / 2 \pi=8.5 \mathrm{MHz}$ ) and 100 GHz-FSR samples (used in ref. ${ }^{26}, \kappa_{0} / 2 \pi=13.5 \mathrm{MHz}$ ) fabricated using the same process but from different wafers. All samples show similar trends, and present two conclusions. First, the mean absorption loss for $40-\mathrm{GHz}-\mathrm{FSR}$ samples is $\kappa_{\mathrm{abs}} / 2 \pi \sim 1 \mathrm{MHz}$, corresponding to an absorption-loss-limited $Q$ factor of approximately $2 \times 10^{8}$. Therefore, the optical losses of our $\mathrm{Si}_{3} \mathrm{~N}_{4}$ waveguides $(\kappa / 2 \pi=6.5 \mathrm{MHz})$ are currently dominated by scattering losses. Second, for all the samples studied, $\kappa_{\mathrm{abs}} / 2 \pi$ is higher around $1520 \mathrm{~nm}$, compared to the value at e.g. $1600 \mathrm{~nm}$. This is caused by the residual hydrogen impurities in our thermally annealed $\mathrm{Si}_{3} \mathrm{~N}_{4}$. Note that only standard LPCVD $\mathrm{Si}_{3} \mathrm{~N}_{4} / \mathrm{SiO}_{2}$ films and thermal annealing are used in our fabrication to achieve such low absorption losses.

\section{Discussion}

In conclusion, we have demonstrated a fabrication technology enabling high-yield and reproducible wafer-scale manufacturing of ultralow-loss, high-confinement, anomalous-GVD $\mathrm{Si}_{3} \mathrm{~N}_{4}$ PIC. This optimized fabrication process employs standard CMOS foundry techniques, and has advantages in multiple aspects compared to previously reported $\mathrm{Si}_{3} \mathrm{~N}_{4}$ fabrication processes (see the comparison chart in the Supplementary Information). We present a statistical study of microresonator losses based on tens of thousands of analyzed resonances. We introduce a novel method to determine the absorption losses, and use it to reveal that our waveguide losses are dominated by scattering losses, which could be further reduced via e.g. optimized lithography and etching. In the ideal case limited only by thermal absorption losses, the potential microresonator $Q$ is calculated to exceed $2 \times$ $10^{8}$ (corresponding to a linear loss of $0.15 \mathrm{~dB} \mathrm{~m}^{-1}$ ). The optimized photonic Damascene fabrication technology allows tight confinement, ultralow-loss, high-yield, meter-scale, nonlinear PIC. Transferring the present $\mathrm{Si}_{3} \mathrm{~N}_{4}$ photonics technology to standard commercial foundries, and merging it with silicon photonics using heterogeneous integration technology ${ }^{3-5}$, will significantly expand the scope of today's integrated photonics and seed new applications.

\section{Methods}

Cavity ring-down. An intensity modulator (IM) is used to rapidly switch off the pump field. The ring-down signal of the transmitted light is recorded by a $1-\mathrm{GHz}$ bandwidth low-noise photodetector. A $50-\mathrm{kHz}$ square wave electrical drive signal is generated using a fast arbitrary waveform generator, ensuring that the light is switched off significantly faster than the resonance linewidth. The upper and lower voltage levels of the square wave are adjusted to match the maximum and minimum transmission voltage of the IM, such that the electrical overshoot and undershoot of the square wave signal do not alter the ring-down slope. Due to the finite extinction ratio of the IM, the residual pump field beats with the leakage of the intracavity field, producing a field ring-down signal which is affected by the detuning of the laser from the cavity mode resonances ${ }^{79}$. At small detunings $(\kappa \gg \Delta)$, the effective ring-down rate is increased by the laser's detuning from cavity resonance, and thus the directly inferred quality factor is less accurate than the sideband fitting result. Therefore, the ring-down results can only serve as a lower bound of the loaded $Q$ factor of the measured resonances. The estimated loaded linewidth $\kappa / 2 \pi=8.4 \mathrm{MHz}$ is in agreement with the sideband fitting results, showing consistency between the three characterization methods used here.

Thermal simulations. We use COMSOL Multiphysics to simulate the thermal response due to bulk absorption heating of our $\mathrm{Si}_{3} \mathrm{~N}_{4}$ waveguides. The main material property coefficients of interest used in the current simulation are identical to the ones used in ref. ${ }^{78}$ for simulating the $\mathrm{Si}_{3} \mathrm{~N}_{4}$ thermorefractive noise. The thermo-optic coefficient ${ }^{80}$ of $\mathrm{Si}_{3} \mathrm{~N}_{4}, \mathrm{~d} n_{\text {mat }} / \mathrm{d} T=2.5 \times 10^{-5} \mathrm{~K}^{-1}$, is used here. We first simulate the waveguide optical mode profile ( $\mathrm{TE}_{00}$ mode), from which the effective mode volume $V_{\text {eff }}$ is calculated. Bulk absorption heating is introduced whose power distribution is proportional to the intensity distribution of the optical mode $v_{m}$. By solving the frequency-domain heat transfer equation, the response of the effective temperature to the modulated absorbed power, $\frac{\mathrm{d} T}{\mathrm{~d} P_{a b s}}(\omega)$, is retrieved from a Fourier frequency sweep. The combined value of $V_{\text {eff }} \cdot \mathrm{d} T / \mathrm{d} P_{\mathrm{abs}}$ is calculated as $3.60 \times 10^{-13} \mathrm{~K} \mathrm{~m}^{3} \mathrm{~W}^{-1}$ in the case of full $\mathrm{SiO}_{2}$ cladding for samples used in Fig. 5e, $\mathrm{f}$, and is $4.63 \times 10^{-13} \mathrm{~K} \mathrm{~m}^{3} \mathrm{~W}^{-1}$ in the case without top $\mathrm{SiO}_{2}$ cladding for samples used in Fig. $5 c$, d.

Response calibration. In order to extract the actual microresonator response $\chi(\omega)$ from the experimentally photodetected $\chi^{\prime}(\omega)$, the frequency response $\chi_{\text {det }}(\omega)$ of our entire experiment setup and detection chain needs to be calibrated first. This is realized by direct detection of the pump power modulation $\delta P(\omega) \propto \chi_{\text {det }}(\omega)$ in the absence of the probe laser and the pump filter. The measured response $\chi^{\prime}(\omega)$ is normalized to the setup response $\chi_{\text {det }}(\omega)$, and thus the actual microresonator response $\chi(\omega)=\chi^{\prime}(\omega) / \chi_{\text {det }}(\omega)$ is retrieved, with a constant factor. This constant factor is removed when retrieving $\chi_{\text {therm }}(0) / \chi_{\text {Kerr }}(0)$ from the fitting of $\chi(\omega)$ using a fitting function

$$
\chi(\omega)=\chi_{\text {Kerr }}(0) \cdot\left(1+\frac{\chi_{\text {therm }}(0)}{\chi_{\text {Kerr }}(0)} \frac{\chi_{\text {therm }}(\omega)}{\chi_{\text {therm }}(0)}\right) \frac{1}{1+2 \mathrm{i} \omega / \kappa_{\text {probe }}} \frac{1}{1+2 \mathrm{i} \omega / \kappa_{\text {pump }}}
$$

with $\kappa_{\text {probe, pump }} / 4 \pi$ being the cavity cutoff frequencies for the pump and probe fields, respectively. The normalized thermal response function $\chi_{\text {therm }}(\omega) / \chi_{\text {therm }}(0)$ used in the fitting is retrieved from COMSOL simulation. In Fig. $5 \mathrm{~d}$, f, only the normalized response $\chi(\omega) / \chi_{\text {Kerr }}(0)$ is shown, with the constant factor removed.

\section{Data availability}

The data that support the plots within this manuscript and other findings of this study are available on Zenodo (https://doi.org/10.5281/zenodo.4273990). All other data used in this study are available from the corresponding authors upon reasonable request.

Received: 17 July 2020; Accepted: 17 February 2021; Published online: 16 April 2021

\section{References}

1. Thomson, D. et al. Roadmap on silicon photonics. J. Opt. 18, 073003 (2016)

2. Agrell, E. et al. Roadmap of optical communications. J. Opt. 18, 063002 (2016)

3. Liang, D., Roelkens, G., Baets, R. \& Bowers, J. E. Hybrid integrated platforms for silicon photonics. Materials 3, 1782-1802 (2010).

4. Park, H., Zhang, C., Tran, M. A. \& Komljenovic, T. Heterogeneous silicon nitride photonics. Optica 7, 336-337 (2020).

5. Blumenthal, D. J. Photonic integration for UV to IR applications. APL Photonics 5, 020903 (2020).

6. Huang, D. et al. High-power sub-kHz linewidth lasers fully integrated on silicon. Optica 6, 745-752 (2019). 
7. Xiang, C. et al. Narrow-linewidth III-V/Si/Si $\mathrm{S}_{3} \mathrm{~N}_{4}$ laser using multilayer heterogeneous integration. Optica 7, 20-21 (2020).

8. Kippenberg, T. J., Gaeta, A. L., Lipson, M. \& Gorodetsky, M. L. Dissipative Kerr solitons in optical microresonators. Science 361, eaan8083 (2018).

9. Gaeta, A. L., Lipson, M. \& Kippenberg, T. J. Photonic-chip-based frequency combs. Nat. Photonics 13, 158-169 (2019).

10. Stern, B., Ji, X., Okawachi, Y., Gaeta, A. L. \& Lipson, M. Battery-operated integrated frequency comb generator. Nature 562, 401-405 (2018).

11. Raja, A. S. et al. Electrically pumped photonic integrated soliton microcomb. Nat. Commun. 10, 680 (2019).

12. Shen, B. et al. Integrated turnkey soliton microcombs. Nature 582, 365-369 (2020).

13. Marin-Palomo, P. et al. Microresonator-based solitons for massively parallel coherent optical communications. Nature 546, 274-279 (2017)

14. Corcoran, B. et al. Ultra-dense optical data transmission over standard fibre with a single chip source. Nat. Commun. 11, 2568 (2020).

15. Obrzud, E. et al. A microphotonic astrocomb. Nat. Photonics 13, 31-35 (2019).

16. Suh, M.-G. et al. Searching for exoplanets using a microresonator astrocomb. Nat. Photonics 13, 25-30 (2019).

17. Trocha, P. et al. Ultrafast optical ranging using microresonator soliton frequency combs. Science 359, 887-891 (2018).

18. Suh, M.-G. \& Vahala, K. J. Soliton microcomb range measurement. Science 359, 884-887 (2018)

19. Riemensberger, J. et al. Massively parallel coherent laser ranging using a soliton microcomb. Nature 581, 164-170 (2020).

20. Spencer, D. T. et al. An optical-frequency synthesizer using integrated photonics. Nature 557, 81-85 (2018).

21. Newman, Z. L. et al. Architecture for the photonic integration of an optical atomic clock. Optica 6, 680-685 (2019).

22. Feldmann, J. et al. Parallel convolutional processing using an integrated photonic tensor core. Nature, 589, 52-58 (2021)

23. $\mathrm{Xu}, \mathrm{X}$. et al. 11 TOPS photonic convolutional accelerator for optical neural networks. Nature, 589, pp. 44-51 (2021).

24. Xuan, Y. et al. High-Q silicon nitride microresonators exhibiting low-power frequency comb initiation. Optica 3, 1171-1180 (2016).

25. Ji, X. et al. Ultra-low-loss on-chip resonators with sub-milliwatt parametric oscillation threshold. Optica 4, 619-624 (2017)

26. Liu, J. et al. Ultralow-power chip-based soliton microcombs for photonic integration. Optica 5, 1347-1353 (2018).

27. Ye, Z., Twayana, K., Andrekson, P. A. \& Torres-Company, V. High-Q $\mathrm{Si}_{3} \mathrm{~N}_{4}$ microresonators based on a subtractive processing for Kerr nonlinear optics. Opt. Express 27, 35719-35727 (2019).

28. Dirani, H. E. et al. Ultralow-loss tightly confining $\mathrm{Si}_{3} \mathrm{~N}_{4}$ waveguides and highQ microresonators. Opt. Express 27, 30726-30740 (2019).

29. Li, Q., Davanço, M. \& Srinivasan, K. Efficient and low-noise single-photonlevel frequency conversion interfaces using silicon nanophotonics. Nat. Photonics 10, 406-414 (2016).

30. $\mathrm{Lu}, \mathrm{X}$. et al. Efficient telecom-to-visible spectral translation through ultralow power nonlinear nanophotonics. Nat. Photonics 13, 593-601 (2019).

31. Hausmann, B. J. M., Bulu, I., Venkataraman, V., Deotare, P. \& Lončar, M. Diamond nonlinear photonics. Nat. Photonics 8, 369-374 (2014).

32. Jung, H. et al. Tantala Kerr-nonlinear integrated photonics. Preprint at http:// arxiv.org/abs/2007.12958 (2020).

33. Guidry, M. A. et al. Optical parametric oscillation in silicon carbide nanophotonics. Optica 7, 1139-1142 (2020).

34. Pu, M., Ottaviano, L., Semenova, E. \& Yvind, K. Efficient frequency comb generation in AlGaAs-on-insulator. Optica 3, 823-826 (2016).

35. Chang, L. et al. Ultra-efficient frequency comb generation in AlGaAs-oninsulator microresonators. Nat. Commun. 11, 1331 (2020).

36. Wilson, D. J. et al. Integrated gallium phosphide nonlinear photonics. Nat. Photonics 14, 57-62 (2020).

37. Wang, C. et al. Integrated lithium niobate electro-optic modulators operating at CMOS-compatible voltages. Nature 562, 101-104 (2018).

38. Zhang, M. et al. Broadband electro-optic frequency comb generation in a lithium niobate microring resonator. Nature 568, 373-377 (2019).

39. He, Y. et al. Self-starting bi-chromatic $\mathrm{LiNbO}_{3}$ soliton microcomb. Optica 6 , 1138-1144 (2019).

40. Fang, Z. et al. Efficient electro-optical tuning of an optical frequency microcomb on a monolithically integrated high-Q lithium niobate microdisk. Opt. Lett. 44, 5953-5956 (2019).

41. Jung, H., Fong, K. Y., Xiong, C. \& Tang, H. X. Electrical tuning and switching of an optical frequency comb generated in aluminum nitride microring resonators. Opt. Lett. 39, 84-87 (2014).

42. Guo, X., Zou, C.-L. \& Tang, H. X. Second-harmonic generation in aluminum nitride microrings with $2500 \% / \mathrm{W}$ conversion efficiency. Optica 3, 1126-1131 (2016).
43. Liu, X. et al. Integrated high-Q crystalline AlN microresonators for broadband Kerr and Raman frequency combs. ACS Photonics 5, 1943-1950 (2018).

44. Moss, D. J., Morandotti, R., Gaeta, A. L. \& Lipson, M. New CMOS-compatible platforms based on silicon nitride and Hydex for nonlinear optics. Nat. Photonics 7, 597-607 (2013).

45. Brasch, V., Chen, Q.F., Schiller, S. \& Kippenberg, T.J. Radiation hardness of high-Q silicon nitride microresonators for space compatible integrated optics. Opt. Express 22, 30786-30794 (2014).

46. Gyger, F. et al. Observation of stimulated Brillouin scattering in silicon nitride integrated waveguides. Phys. Rev. Lett. 124, 013902 (2020).

47. Brasch, V. et al. Photonic chip-based optical frequency comb using soliton Cherenkov radiation. Science 351, 357-360 (2016).

48. Kovach, A. et al. Emerging material systems for integrated optical Kerr frequency combs. Adv. Opt. Photonics 12, 135-222 (2020).

49. Spencer, D. T., Bauters, J. F., Heck, M. J. R. \& Bowers, J. E. Integrated waveguide coupled $\mathrm{Si}_{3} \mathrm{~N}_{4}$ resonators in the ultrahigh-Q regime. Optica 1, 153-157 (2014).

50. Gundavarapu, S. et al. Sub-hertz fundamental linewidth photonic integrated Brillouin laser. Nat. Photonics 13, 60-67 (2019).

51. Bauters, J. F. et al. Planar waveguides with less than $0.1 \mathrm{~dB} / \mathrm{m}$ propagation loss fabricated with wafer bonding. Opt. Express 19, 24090-24101 (2011).

52. Guo, H. et al. Nanophotonic supercontinuum-based mid-infrared dual-comb spectroscopy. Optica, 7, 1181-1188 (2020).

53. Baumann, E. et al. Dual-comb spectroscopy with tailored spectral broadening in $\mathrm{Si}_{3} \mathrm{~N}_{4}$ nanophotonics. Opt. Express 27, 11869-11876 (2019).

54. Selvaraja, S. K. et al. Highly uniform and low-loss passive silicon photonics devices using a 300mm CMOS platform. In Optical Fiber Communication Conference, OSA Technical Digest (online), paper Th2A.33 (Optical Society of America, 2014) https://www.osapublishing.org/abstract.cfm?uri=OFC-2014Th2A.33.

55. Ciminelli, C., Dell'Olio, F., Armenise, M. N., Soares, F. M. \& Passenberg, W High performance InP ring resonator for new generation monolithically integrated optical gyroscopes. Opt. Express 21, 556-564 (2013).

56. Foster, M. A. et al. Broad-band optical parametric gain on a silicon photonic chip. Nature 441, 960-963 (2006).

57. Kuyken, B. et al. $50 \mathrm{~dB}$ parametric on-chip gain in silicon photonic wires. Opt. Lett. 36, 4401-4403 (2011).

58. Ooi, K. J. A. et al. Pushing the limits of CMOS optical parametric amplifiers with USRN:Si $\mathrm{N}_{3}$ above the two-photon absorption edge. Nat. Commun. 8 , 13878 (2017).

59. Yang, M. et al. An octave-spanning optical parametric amplifier based on a low-dispersion silicon-rich nitride waveguide. IEEE J. Sel. Top. Quantum Electron. 24, 1-7 (2018)

60. Xin, M. et al. Optical frequency synthesizer with an integrated erbium tunable laser. Light: Sci. Appl. 8, 122 (2019).

61. Pfeiffer, M. H. P. et al. Photonic Damascene process for low-loss, highconfinement silicon nitride waveguides. IEEE J. Sel. Top. Quantum Electron. 24, 1-11 (2018)

62. Wu, K. \& Poon, A. W. Stress-released $\mathrm{Si}_{3} \mathrm{~N}_{4}$ fabrication process for dispersion-engineered integrated silicon photonics. Opt. Express 28, 17708-17722 (2020)

63. Pfeiffer, M. H. P. et al. Ultra-smooth silicon nitride waveguides based on the Damascene reflow process: fabrication and loss origins. Optica 5, 884-892 (2018).

64. Liu, J. et al. Monolithic piezoelectric control of soliton microcombs. Nature 583, 385-390 (2020).

65. Tian, H. et al. Hybrid integrated photonics using bulk acoustic resonators. Nat. Commun. 11, 3073 (2020)

66. Churaev, M. et al. Hybrid $\mathrm{Si}_{3} \mathrm{~N}_{4}-\mathrm{LiNbO}_{3}$ integrated platform for electro-optic conversion. In Conference on Lasers and Electro-Optics, STh1F.3 (Optical Society of America, 2020) https://www.osapublishing.org/abstract.cfm? uri=CLEO SI-2020-STh1F.3.

67. He, J. et al. Low-Loss Integrated Nanophotonic Circuits with Layered Semiconductor Materials, Nano Letters. https://doi.org/10.1021/acs. nanolett.0c04149.

68. Luke, K., Okawachi, Y., Lamont, M. R. E., Gaeta, A. L. \& Lipson, M. Broadband mid-infrared frequency comb generation in a $\mathrm{Si}_{3} \mathrm{~N}_{4}$ microresonator. Opt. Lett. 40, 4823-4826 (2015).

69. Poon, J. K. S., Scheuer, J., Xu, Y. \& Yariv, A. Designing coupled-resonator optical waveguide delay lines. J. Opt. Soc. Am. B 21, 1665-1673 (2004).

70. Cardenas, J. et al. Wide-bandwidth continuously tunable optical delay line using silicon microring resonators. Opt. Express 18, 26525-26534 (2010).

71. Del'Haye, P., Arcizet, O., Gorodetsky, M. L., Holzwarth, R. \& Kippenberg, T. J. Frequency comb assisted diode laser spectroscopy for measurement of microcavity dispersion. Nat. Photonics 3, 529-533 (2009).

72. Liu, J. et al. Photonic microwave generation in the X-and K-band using integrated soliton microcombs. Nat. Photonics 14, 486-491 (2020). 
73. Li, J., Lee, H., Yang, K. Y. \& Vahala, K. J. Sideband spectroscopy and dispersion measurement in microcavities. Opt. Express 20, 26337-26344 (2012).

74. Lee, H., Chen, T., Li, J., Painter, O. \& Vahala, K. J. Ultra-low-loss optical delay line on a silicon chip. Nat. Commun. 3, 867 (2012).

75. Ji, X. et al. On-chip tunable photonic delay line. APL Photonics 4, 090803 (2019).

76. Griffith, A., Cardenas, J., Poitras, C. B. \& Lipson, M. High quality factor and high confinement silicon resonators using etchless process. Opt. Express 20, 21341-21345 (2012)

77. Kim, D. G. et al. Universal light-guiding geometry for on-chip resonators having extremely high Q-factor. Nat Commun 11, 5933 (2020).

78. Huang, G. et al. Thermorefractive noise in silicon-nitride microresonators. Phys. Rev. A 99, 061801 (2019).

79. Wójtewicz, S. et al. Response of an optical cavity to phase-controlled incomplete power switching of nearly resonant incident light. Opt. Express 26, 5644-5654 (2018).

80. Arbabi, A. \& Goddard, L. L. Measurements of the refractive indices and thermo-optic coefficients of $\mathrm{Si}_{3} \mathrm{~N}_{4}$ and $\mathrm{SiO}_{x}$ using microring resonances. Opt. Lett. 38, 3878-3881 (2013).

\section{Acknowledgements}

The authors thank Qi-Fan Yang, Maodong Gao, and Kerry Vahala for the critical suggestion to the data analysis of thermal response. We thank Bahareh Ghadiani for the assistance in the fabrication process development in the early stage. This work was supported by Contract HR0011-15-C-055 (DODOS) from the Defense Advanced Research Projects Agency (DARPA), Microsystems Technology Office (MTO), by the Air Force Office of Scientific Research, Air Force Materiel Command, USAF under Award No. FA9550-15-1-0250, and by Swiss National Science Foundation under grant agreement No. 176563 (BRIDGE). G.H. and N.J.E. acknowledge support from the Swiss National Science Foundation under grant no. 185870 (Ambizione). The $\mathrm{Si}_{3} \mathrm{~N}_{4}$ microresonator samples were fabricated in the EPFL center of MicroNanoTechnology (CMi).

\section{Author contributions}

J.L. developed the fabrication process with the assistance from R.N.W. J.L. and R.N.W. fabricated the $\mathrm{Si}_{3} \mathrm{~N}_{4}$ samples. J.H., J.L., G.H., A.S.R., T.L., and N.J.E characterized the samples and analyzed the data. G.H., N.J.E., and J.L. performed the microresonator response measurement and analyzed the data. J.L., G.H., and T.J.K. wrote the manuscript, with the input from others. T.J.K. supervised the project.

\section{Competing interests}

The authors declare no competing interests.

\section{Additional information}

Supplementary information The online version contains supplementary material available at https://doi.org/10.1038/s41467-021-21973-Z

Correspondence and requests for materials should be addressed to T.J.K.

Peer review information Nature Communications thanks Andrea Armani and the other anonymous reviewers for their contribution to the peer review of this work. Peer reviewer reports are available.

Reprints and permission information is available at http://www.nature.com/reprints

Publisher's note Springer Nature remains neutral with regard to jurisdictional claims in published maps and institutional affiliations.

Open Access This article is licensed under a Creative Commons Attribution 4.0 International License, which permits use, sharing, adaptation, distribution and reproduction in any medium or format, as long as you give appropriate credit to the original author(s) and the source, provide a link to the Creative Commons license, and indicate if changes were made. The images or other third party material in this article are included in the article's Creative Commons license, unless indicated otherwise in a credit line to the material. If material is not included in the article's Creative Commons license and your intended use is not permitted by statutory regulation or exceeds the permitted use, you will need to obtain permission directly from the copyright holder. To view a copy of this license, visit http://creativecommons.org/ licenses/by/4.0/

(C) The Author(s) 2021 